\title{
EXPERIMENTAL STUDY OF A WIND-POWERED HEAT GENERATOR WITH NANOFLUIDS AGITATION
}

\author{
Muhammad Haseeb Javed, Xili Duan \\ Faculty of Engineering and Applied Sciences, \\ Memorial University of Newfoundland, St John's, Newfoundland, Canada
}

\begin{abstract}
Modern wind turbines are generally used for generation of electricity, however the final form of energy required by user in many cases is thermal energy. Although conversion of electrical energy to thermal energy is a high efficiency process but the efficiency of electricity generation from wind turbines is usually low. We proposed a novel direct wind thermal energy conversion (WinTEC) device that converts the kinetic energy from wind directly into thermal energy through the process of viscous dissipation which is achieved through agitation of a working fluid in a container. Water based Aluminum oxide nanofluid is used as working fluid because of its superior thermal fluid properties. This WinTEC device uses an optimized flat blade turbine (FBT) with a baffled design. We use four standard baffles each $10 \%$ the diameter of the cylindrical container for maximum power dissipation. An electric motor is used to provide the mechanical input and a torque sensor and tachometer are used to measure the efficiency of the heat generator in converting this mechanical energy into thermal energy. Experiments are conducted at different rotational speeds and for different working fluids: distilled water and nanofluid. Our results indicate that the rate of temperature rise increases for higher rotational speed and greater nanoparticle concentration. The device will be scalable to fit the size and need of a house or a commercial building. This innovative renewable energy technology would have a beneficial impact on the economic prosperity, environmental sustainability, and social well-being in many regions of the world, particularly, in remote cold regions with rich wind energy resources.
\end{abstract}

Word count: 259 